Association for Information Systems AIS Electronic Library (AISeL)

Wirtschaftsinformatik Proceedings 2001

Wirtschaftsinformatik

September 2001

\title{
IT in der Medienindustrie - Trends und Anforderungen
}

Daniel Hartert

Bertelsmann mediaSystems, daniel.hartert@bertelsmann.de

Follow this and additional works at: http://aisel.aisnet.org/wi2001

\section{Recommended Citation}

Hartert, Daniel, "IT in der Medienindustrie - Trends und Anforderungen" (2001). Wirtschaftsinformatik Proceedings 2001. 6. http://aisel.aisnet.org/wi2001/6

This material is brought to you by the Wirtschaftsinformatik at AIS Electronic Library (AISeL). It has been accepted for inclusion in Wirtschaftsinformatik Proceedings 2001 by an authorized administrator of AIS Electronic Library (AISeL). For more information, please contact elibrary@aisnet.org. 
In: Buhl, Hans Ulrich, u.a. (Hg.) 2001. Information Age Economy; 5. Internationale Tagung Wirtschaftsinformatik 2001. Heidelberg: Physica-Verlag

ISBN: 3-7908-1427-X

(C) Physica-Verlag Heidelberg 2001 


\title{
IT in der Medienindustrie - Trends und Anforderungen
}

\author{
Daniel Hartert \\ Bertelsmann mediaSystems
}

\begin{abstract}
Zusammenfassung: In keiner andere Branche gibt es eine derart öffentliche und hart geführte Debatte über zukünftige Geschäftsmodelle wie in Medien- und Unterhaltungsindustrie. Die potentiellen Veränderungen durch Peer-to-Peer-Netze und durch E-Business und durch neue Endgeräte führen zu einem radikalen Umbruch in der gesamten Branche. Alle diese Entwicklungen sind technologiegerieben, und somit ist für Medienunternehmen neben dem Produzieren von Inhalten Technologie der Schlüsselerfolgsfaktor geworden. Der Beitrag erläutert die Adaption neuer Technologietrends in die Geschäftsmodelle von Medienunternehmen. Insbesondere beleuchtet er die Verbindung zwischen der Konvergenztechnologie und einem erfolgreichen E-Commerce von Inhalten.
\end{abstract}

Daniel Hartert ist verantwortlich für Bertelsmann mediaSystems, dem größten ITDienstleister im Bertelsmann Konzern. Die Konvergenzlösungen von mediaSystems spielen eine wichtige Rolle in der Vorbereitung der Kunden für das Zeitalter digitaler Medien.

Schlüsselworte: Medienindustrie, Geschäftsmodelle, Wertschöpfungskette, Mobile Communication, E-Commerce Distribution, Bündelung, Konvergenz, IT-Systeme

\section{Die klassische Wertschöpfung in der Medienindustrie}

Nahezu alle Zweige der Branche Medienindustrie stehen derzeit vor der Herausforderung, angemessen auf die neuen technologischen Möglichkeiten und die damit verbundenen Wettbewerber und Geschäftsmodelle zu reagieren. So eröffnen beispielsweise File-Sharing-Tools, wie Napster in der Musikindustrie oder EBooks im Verlagsbereich, völlig neue Vertriebswege, die einerseits das angestammte Geschäft bedrohen, anderseits aber auch Chancen in neuen Märkten bieten.

Diese ,,angestammten Geschäfte“ lassen sich an der klassischen Wertschöpfungskette der Medienindustrie und ihren einzelnen Prozessstufen definieren (Abb. 1). Sie wurden und werden im Allgemeinen durch ausgefeilte IT-Systeme unterstützt, 
die, ähnlich den ERP-Systemen in der Industrie, explizit auf das Business der Medienindustrie zugeschnitten wurden.

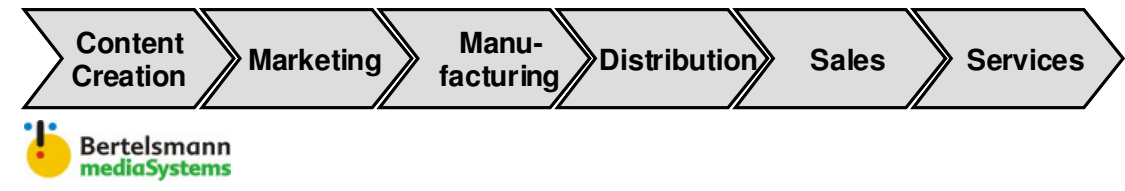

Abbildung 1: Die Wertschöpfungskette der Medienindustrie und ihre Ausprägungen (in Anlehnung an [Rayp99], S. 39)

Am Beispiel der Musikindustrie kann die o. a. Wertschöpfungskette deutlich gemacht werden. In der ersten Stufe Content Creation werden Künstler gesucht, Titel erdacht und in Tonstudios aufgenommen sowie geschnitten. Im nächsten Schritt Marketing erfolgt die Bewerbung des Titel-Bündels ${ }^{1}$ in verschiedenen Medien und mit diversen Promotion-Aktionen. Mit der Herstellung und Verteilung werden die Titel-Bündel auf ein Medium gepresst und zu den Stellen verteilt, an denen der Endkonsument dieses dann erwerben bzw. ausleihen kann. Der Service schließlich ermittelt Kundendaten um zukünftige Produkte gezielter herzustellen und umfasst weitere Verkaufsaktionen von Cross-Selling-Produkten.

Was hat das alles mit IT zu tun? Keine der Stufen kann ohne spezielle Systeme auskommen. Am Beispiel der Musikindustrie: Neben den Recording- und Nachbearbeitungsgeräten im Tonstudio sind hier die klassischen Industrielösungen wie SCM-/PPS-Systeme und Vertriebsunterstützungssysteme sowie ausgefeilte Rechtemanagement-Tools erforderlich.

\section{Einfluss neuer Technologien auf die Wertschöpfung}

\subsection{Zukünftige Schlüsseltechnologien}

Die Diskussion über zukünftige Schlüsseltechnologien für die Medienindustrie kann und muss losgelöst von deren Umsetzbarkeit in profitable Geschäftsmodelle geführt werden, da die Technologien im Allgemeinen nicht durch die Industrie forciert, sondern von Anwendern nachgefragt und eingesetzt werden. Hier kann das Beispiel Napster angeführt werden: Mit dem Dienst wurde die P2P-Technologie etabliert, weil es für die Anwender bequem und kostengünstig ist, privat qualitativ hochwertige Musik auszutauschen. Die Medienindustrie konnte den

${ }^{1}$ Z. B. eine CD. 
einmal gestarteten Zug nicht mehr aufhalten. Schlüsseltechnologien sind somit technologische Entwicklungen, die Voraussetzungen für die Anwendung neuer Geschäftsmodelle sind - egal, ob erfolgreich oder nicht. Deshalb kann auch das Synonym „Enabling Technologies“ Verwendung finden.

Rückblickend etablierte sich die Digitalisierung als die Schüsseltechnologie für die Medienindustrie. Sie ermöglichte zunächst die verlustfreie und kostengünstigere Speicherung von qualitativ hochwertigen Inhalten aller Medien (Abb. 2). Dann kamen Systeme zur verlustfreien Vervielfältigung und Transport der Inhalte hinzu, so dass die Vorteile der Digitalisierung beim Endkunden auch ankamen.

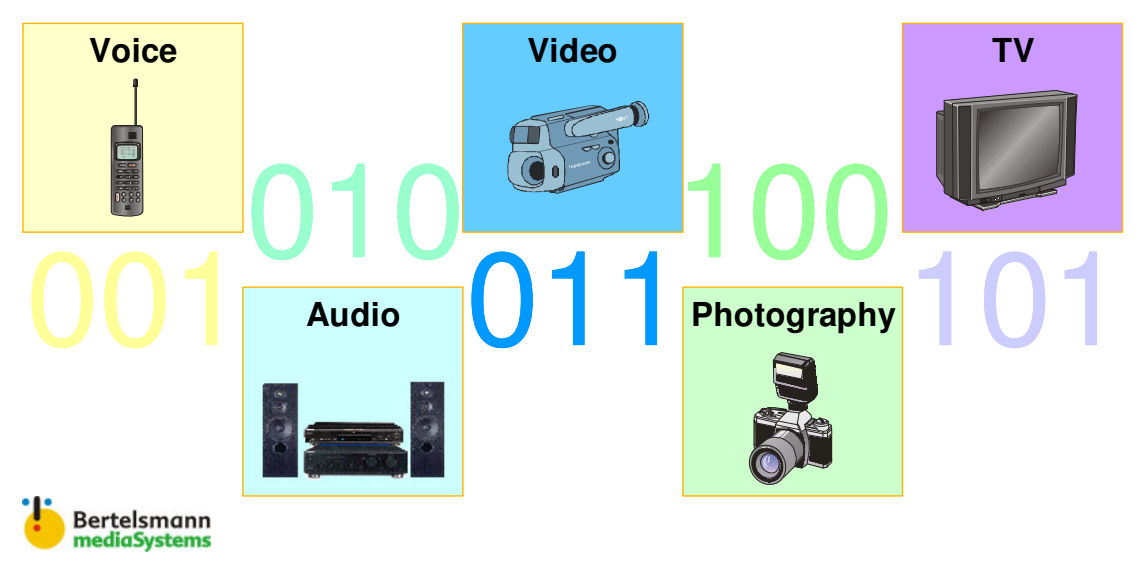

Abbildung 2: Digitalisierung der Inhalte

Die Digitalisierung ist heute die Basis für den Erfolg anderer Schlüsseltechnologien. Deshalb werden sie im Folgenden als sekundäre Schlüsseltechnologien bezeichnet. Hier lassen sich drei Bereiche identifizieren, die wesentlichen Einfluss auf die Geschäftsmodelle der Medienindustrie besitzen (Abb. 3): Die Entwicklung der Informationstechnologie selbst als erste Enabling Technology ermöglicht mit den drei Elementen Kompression, Speicherkapazität und Rechengeschwindigkeit die kostengünstige Speicherung, Bearbeitung und vor allem den individuellen Abruf von Inhalten. Die Telekommunikation als zweiter Treiber ermöglicht mit Breitbandzugängen den schnellen und ebenfalls kostengünstigen, nicht physischen Transport von Inhalten ([VonB97], S. 98 f.). Und schließlich ermöglichen mobile und stationäre Endgeräte die Speicherung und den Konsum der Inhalte. Somit sind aus Sicht der Konsumenten alle digitalisierten Medien jederzeit, überall und individuell gebündelt abrufbar. 


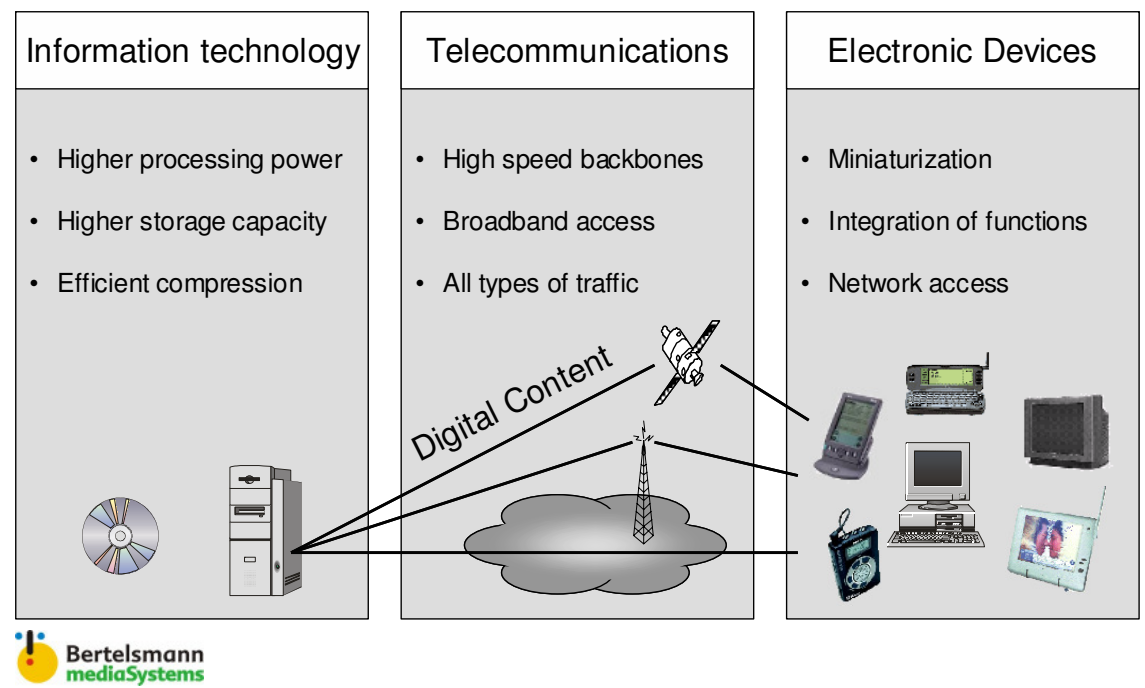

Abbildung 3: Sekundäre Schlüsseltechnologien

Die Integration der drei sekundären Schlüsseltechnologien mit den medialen Inhalten führt zu dem Megatrend Konvergenz. Konnten sich gestern noch Konzerne um jeweils eine Schlüsseltechnologie etablieren (Abb. 4), so zwingt in Zukunft die Konvergenz die Unternehmen sehr viel stärker zusammenzuarbeiten. Hier können zahlreiche Beispiele angeführt werden, die auch die Bedeutung neuer Geschäftsmodelle für die drei Branchen ${ }^{2}$ unterstreichen: So fokussiert sich Deutschlands größter Internet-Provider T-Online zunehmend auf den Content-Bereich und AOL fusionierte mit Time-Warner. Aktuell stehen die Betreiber der zukünftigen UTMS-Netzwerke vor dem Problem, die breitbandigen Datenzugänge zu den Endgeräten mit entsprechenden (durch Kunden nachgefragte und bezahlte) Inhalten auszunutzen.

\footnotetext{
${ }^{2}$ Die Schnittmenge aller drei Branchen bilden die Konvergenz-Branche.
} 


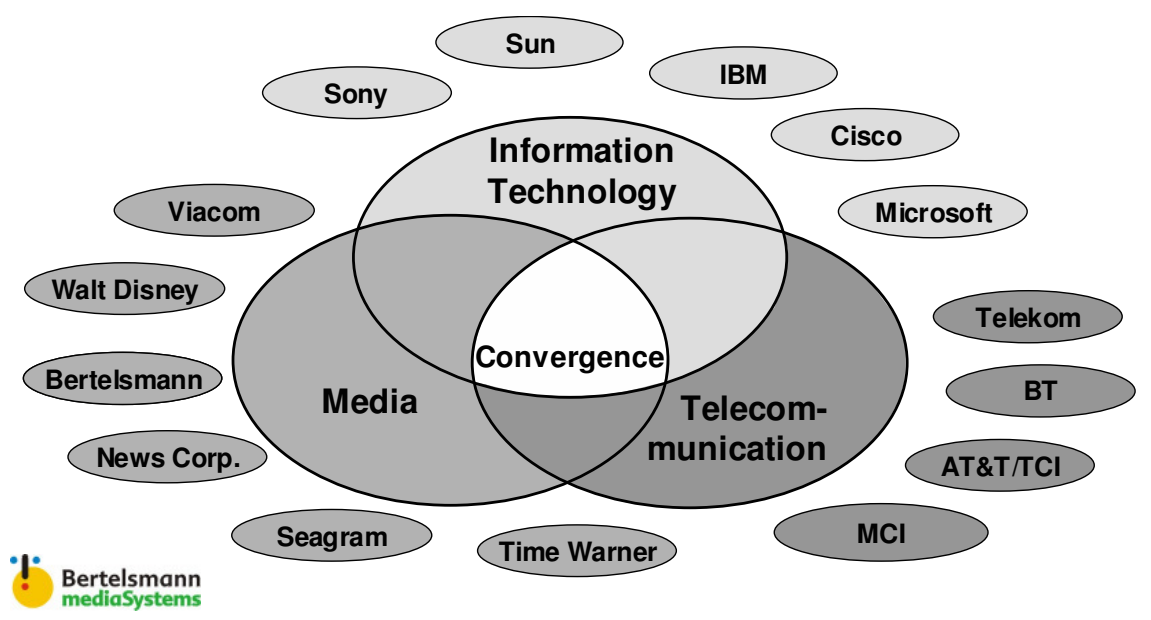

Abbildung 4: Konvergenz

\subsection{Die Wertschöpfungskette der Medienindustrie im Umbruch}

Zur Betrachtung der technologiegetriebenen Veränderungen in der Medienindustrie ist es notwendig, die einzelnen Prozessstufen der Wertschöpfungskette aus Abbildung 1 genauer zu analysieren. Der erste Schritt - die Erstellung der Inhalte - wurde bereits vor ca. 15-20 Jahren durch die Digitalisierung revolutioniert, die entsprechenden Technologien sind deshalb etabliert und tragen zu keiner zukünftigen weiteren Veränderung der Geschäftsmodelle bei. Deshalb wird an dieser Stelle nicht weiter auf diese Prozessstufe eingegangen.

Das Marketing als zweites Element der Wertschöpfungskette unterliegt dagegen derzeit einem starken Wandel - weg von der Massenansprache hin zu der individuellen Bewerbung gezielter Personen, deren Kaufverhalten einen exakten Fit mit angebotenen Inhalten hat ([Wolf99] S. 82 ff.). Unter den Stichworten „One-toone-Marketing“ und „Customer-Profiling“ versuchen die Medienunternehmen zunächst das Verhalten der Kunden zu ermitteln um diese Informationen auszunutzen, maßgeschneiderte Angebote für den einzelnen Kunden oder Kundengruppen zu entwickeln (Abb. 5). Hier sind auf die Medienindustrie abgestimmte CRMSysteme die absolute Grundvoraussetzung (s. u.). 


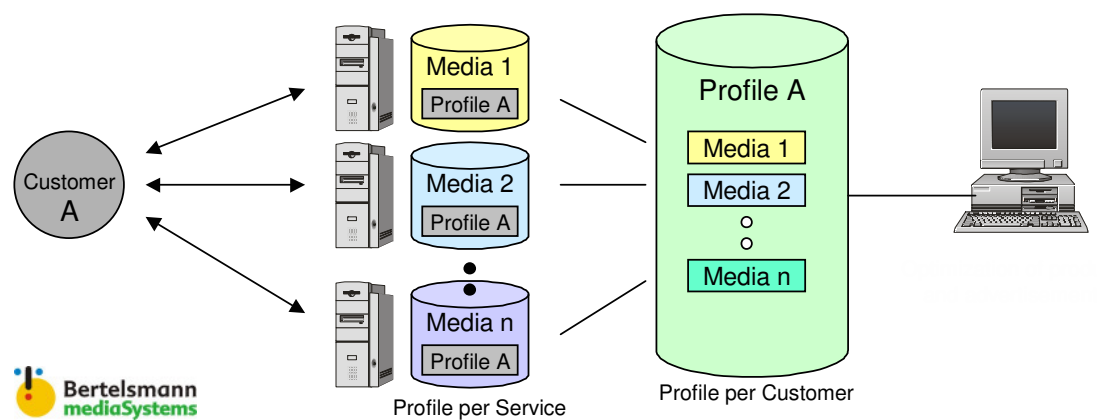

Abbildung 5: Zukünftiges Marketing in der Medienindustrie

Der Schritt der Herstellung von Datenträgern entfällt im Bereich der Medienindustrie zunehmend und wird ersetzt durch die medienlose, also nicht-physische Zusammenstellung von Inhalten. Diese Bündelung erfolgt im Allgemeinen nach dem Pull-Prinzip. Der Kunde ist also selber aktiv. Die nicht-physische Bündelung ermöglicht ganz neue Konzepte: Digitalisierte Inhalte können durch den Kunden und / oder systemunterstützt beliebig, medienübergreifend und nach bestimmten Zielsetzungen zusammengestellt werden ([Schw99], S. 113 ff.). Ein Beispiel soll dieses verdeutlichen (Abb. 6): Möchte ein Kunde einen italienischen Abend genießen, kann er sich auf Knopfdruck ein Komplettpaket aus Kochbuch, Musik, Film und Reiseinformationen zusammenstellen. Dazu sind aber medienübergreifende Content- und Rechtemanagement-Tools notwendig.

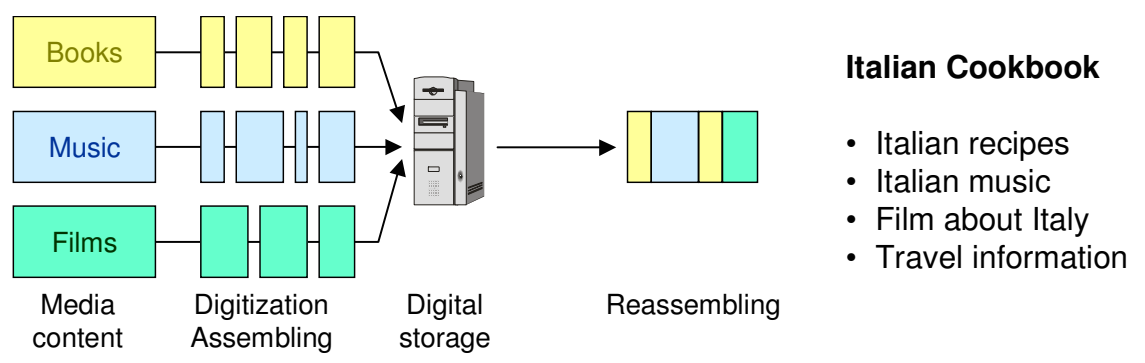

$\because$ Bertelsmann

Abbildung 6: Zukünftige Fertigung der Inhalte in der Medienindustrie

Solche Inhalte können dann zwar für den Kunden individuell hergestellt werden ${ }^{3}$; schneller, kostengünstiger und einfacher ist aber die nicht-physische Distribution der digitalisierten Inhalte als Datenpakete direkt zum Endgerät des Kunden. Letz-

${ }^{3}$ Das ist z. B. beim Print-On-Demand der Fall. 
teres ist beliebig, solange Standards unterstützt werden (Abb. 7). Der Prozess aus Bündelung und Distribution verläuft integriert ab und erfordert ein enges Zusammenspiel von Inhaltslieferant und Network Provider, da der Kunde in einer Plattform arbeiten sollte.

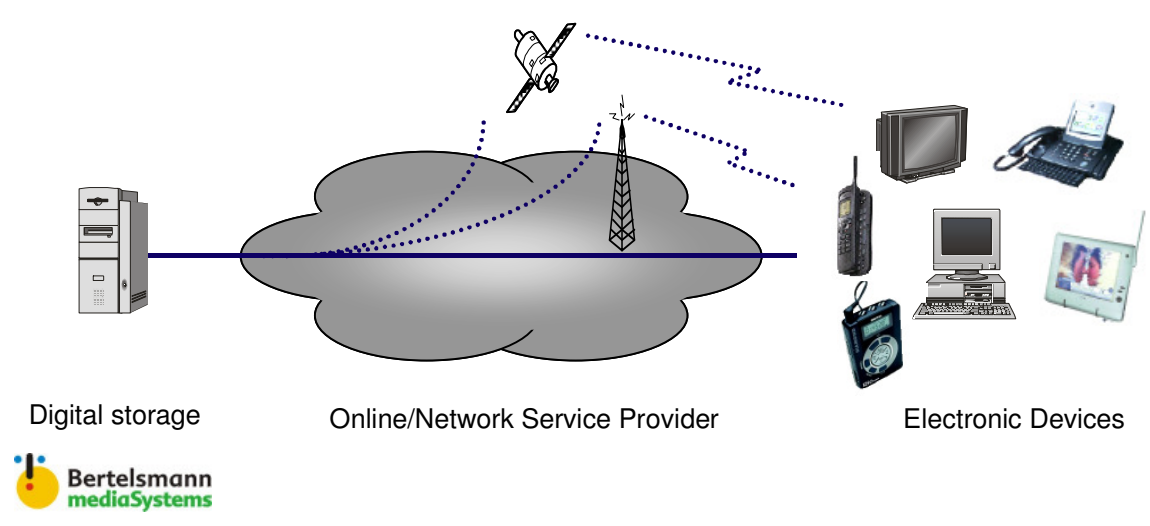

Abbildung 7: Zukünftige Distribution der Inhalte in der Medienindustrie

Die Abrechnung von zum Kunden gebrachten Inhalten wird in der jetzigen Phase der New Economy zunehmend ein Thema. Ist ein Kunde bereit, für einen Inhalt Geld zu bezahlen, dann muss einerseits das Geschäftsmodell von einem werbefinanzierten Dienst auf ein Rechnungsmodell umgestellt werden. Das impliziert aber andererseits auch eine hohe Integration in sogenannte Back-End-Systeme, die die dann wesentlich komplexere Prozesse unterstützen (Abb. 8).

Die notwendigen Transaktionssysteme können nicht von der Stange gekauft und eingeführt werden, sondern erfordern weitreichende unternehmensspezifische Anpassungen. Beim so entstehenden E-Commerce gibt es zunehmend Probleme mit dem Bezahlverhalten der Kunden. Das ist ein Grund, warum zunehmend Unternehmen das E-Commerce inklusive das Backend, die Produktbereitstellung und das Finance-Clearing zu einem Dienstleister outsourcen. 


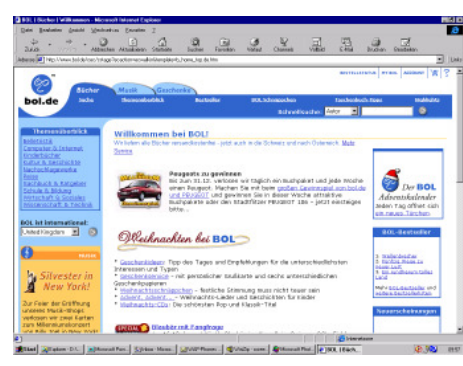

Web Front End

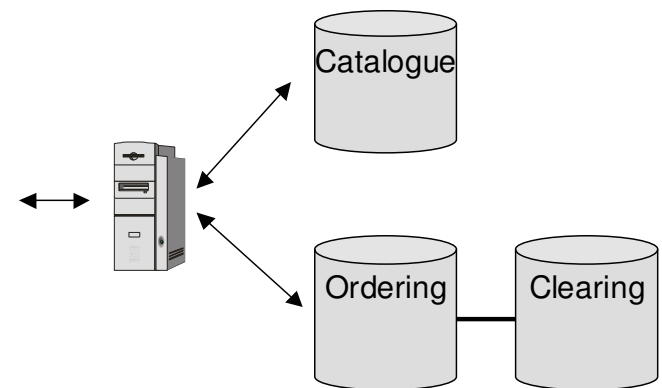

Back End

\section{B. Bertelsmann}

Abbildung 8: Zukünftiger Verkauf der Inhalte in der Medienindustrie

Mit dem Service schließt sich der Kreis zum Marketing, da durch den Service wesentliche Informationen über den Kunden gewonnen werden. So kann die durch den Kunden durchgeführte Personalisierung ausgenutzt werden, dezidierte Kundenprofile zu erstellen (Abb. 9, vgl. [Nais86], S. 61 f.). Zudem bindet die Personalisierung den Kunden an das Unternehmen bzw. den Service. CRM ist auch hier das Schlagwort, unter dem die notwendige Systemunterstützung zusammengefasst wird.
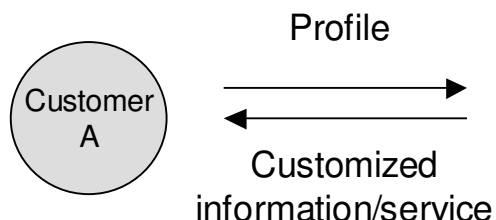

Bertelsmann
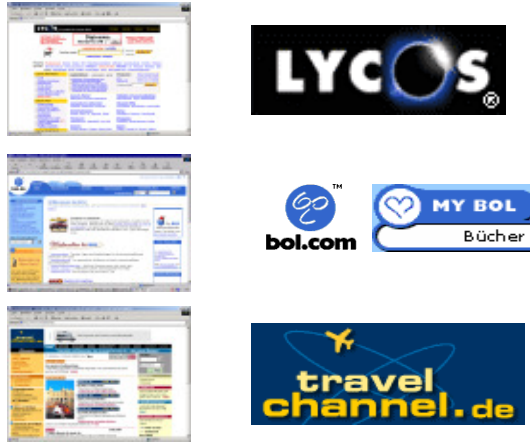

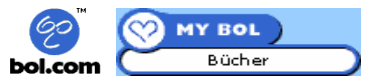

Abbildung 9: Zukünftiger Service in der Medienindustrie

\subsection{Beispiel für geänderte Business-Modelle - Interactive TV}

Das Interactive TV hat den Ansatz von Fernsehen als unidirektionalen Kanal zum Konsumenten obsolet gemacht und in ein bidirektionales Modell umgewandelt (Abb. 10). Wurde mit Kabelnetzen, Satellitenübertragung und Digital TV in den 
vergangenen Jahren die Vielfalt der einem Empfänger bereitgestellten Programme ständig erhöht, so verfolgt das Interactive TV den Ansatz, dem Konsumenten nur noch ein Programm bereitzustellen, das er sich dann aber selbst zusammenstellen kann. Der übertragene Inhalt ${ }^{4}$ wird angereichert mit Zusatzdiensten, die ebenfalls auf das aktuelle Konsumbedürfnis des Kunden abgestimmt sind und zusätzlich Geschäfte generieren. Die resultierenden Revenue-Streams setzen sich also aus Grundgebühren, Pay-Per-View, E-Commerce und Werbung zusammen.

Auf der anderen Seite sind Investitionen in die derzeit nicht vorhandene Infrastruktur und Systemunterstützung notwendig. Zudem müssen die Unternehmen der Medien-, IT- und Telekommunikationsbranche (Abb. 4) die Konvergenz meistern und Wege finden, Investitionen, Einnahmen und Systeme zielführend zu teilen. Dennoch wird dem Interactive TV eine große Zukunft vorausgesagt.

\section{Broadcast}

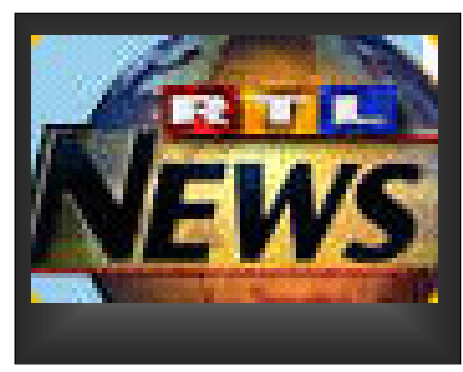

- Uni-directional

- Mass medium

- Fixed time slot

- Videotext

- No communication

- No shopping

?'Bertelsmann

Abbildung 10: Beispiel Interactive TV

\section{Interactive}

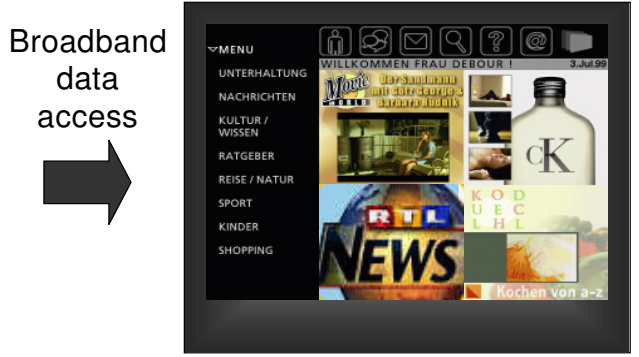

- Bi-directional

- Personalization

- On demand

- Electronic Programming Guide

- E-mail

- E-Commerce

${ }^{4}$ Z. B. ein Film oder eine Dokumentation. 


\section{Herausforderungen für die IT in der Medienindustrie}

Die Schlüsseltechnologien sind weitgehend ausgereift und werden von den verschiedenen Dienstleistern der IT-, Medien- und Telekommunikationsbranche angeboten. Für das einzelne Medienunternehmen stellen sich bei der Implementierung der neuen Geschäftsmodelle aber einige Probleme, die zusätzlicher Systemunterstützung bedürfen. Hier seien zwei Beispiele herausgegriffen, die derzeit noch nicht vollständig gelöst sind.

Die Systeme zum Digital Rights Management (DRM) basierten früher auf dezidierte Autorenverträge, die für jedes Land explizit vorschreiben, wie, auf welchem Medium, und in welchen Bündeln der Inhalt vertrieben werden darf und welche Tantiemen dafür zu bezahlen sind ([Choi97], S. 175 ff.). Mit der o. a. freien Zusammenstellung und der weltweiten möglichen Distribution von Inhalten müssen solche Regeln bei jeder Transaktion überprüft werden. Das beschränkt einerseits die Freiheit des Konsumenten stark und anderseits wird eine solche Transaktion sehr komplex und langwierig. Die Systemunterstützung muss entsprechend ausgebaut sein und wird somit sehr kostenintensiv. Viele Vorteile der Schlüsseltechnologien sind somit für den Konsumenten nicht mehr vorhanden ([Hage97], S. 174). Deshalb ist an zwei Stellen anzusetzen. Zum einen müssen die Verträge mit den Künstlern die neuen Distributionswege zulassen. Gebiets- und Bündelungsbeschränkungen dürfen nicht für das digitale Anbieten von Inhalten gelten. Zum anderen müssen die Medienunternehmen DRM-Dienste bereitstellen, mit denen Anbietersysteme eine Rechtekontrolle durchführen können. Heutige Plattformen zum Vertrieb von Medien decken das DRM nicht oder nicht ausreichend ab.

Als zweites Beispiel soll die Rechnungsstellung zum Konsumenten dienen. Nicht jeder Inhalt kann kostenlos angeboten werden. Da aber die individuelle Bündelung von Inhalten auch die individuelle Rechnungsstellung von kleinsten Einheiten ${ }^{5}$ erfordert, ist das Micro-Payment eine zwingende Voraussetzung für die Umsetzung der Schlüsseltechnologien in erfolgreiche Geschäftsmodelle. Micro-Payment erfordert aber anderseits geringste Transaktionskosten im Rechnungsstellungsprozess, so dass hier völlig automatisierte Dienste wie Guthabenabbuchungen, EWallets, Abogebühren oder Abrechnungsaufschläge auf Telefongebühren eingeführt werden müssen. Sie alle sind derzeit in der Entwicklung, etabliert haben sie sich noch nicht.

Weitere Beispiele für notwendige IT-Systeme sind die Integration von Geschäftspartnern der Konvergenz-Branche, das in die Wertschöpfung integrierte und medienübergreifende Customer Relationship Management, die Vereinheitlichung von Komprimierungs- und Speicherstandards digitaler Inhalte und das Agieren bzw. Reagieren auf sich ständig ändernde Konsumbedürfnisse (Abb. 11, vgl. [Cusu98],

${ }^{5}$ Z. B. ein Musikstück oder ein Nachrichtenartikel. 
S. 156 ff.). Hier entsteht ein Trade-Off zwischen Systemkomplexität und -investitionen einerseits und Integration und Kundenbedürfnisbedienung andererseits. Jedes Unternehmen der Medienindustrie muss in diesem Trade-Off für sich ein Optimum herstellen und mit seinem IT-Dienstleister die notwendigen Systeme aufbauen.

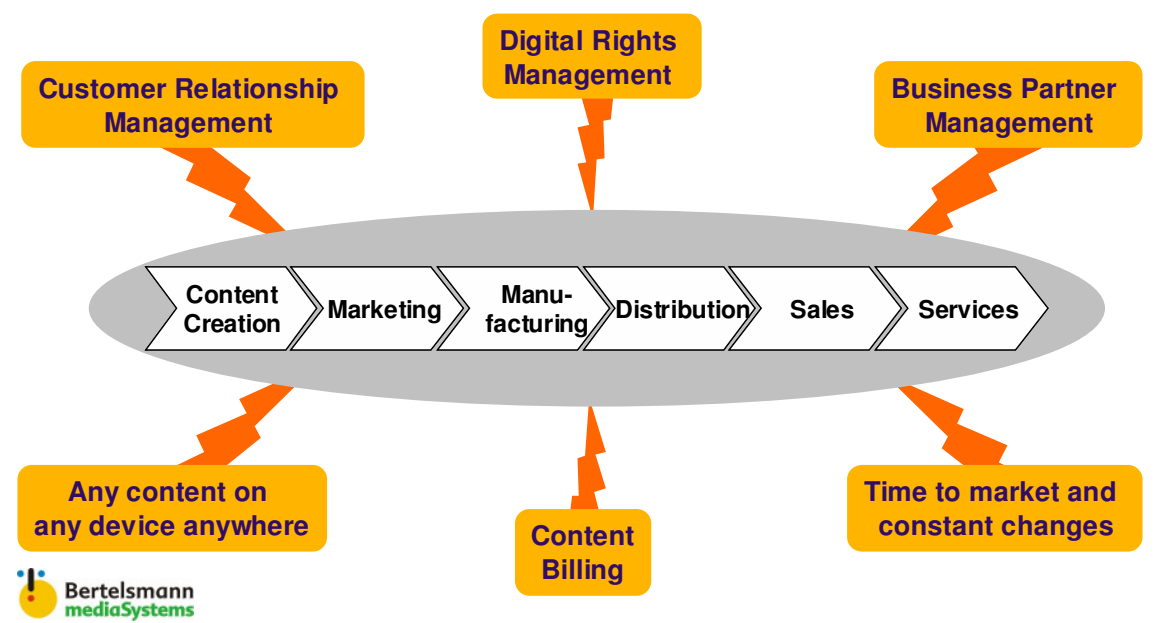

Abbildung 11: Informationstechnologische Herausforderungen der Medienindustrie

\section{Ausblick}

In der Zukunft werden die technologiegetriebenen Geschäftsmodelle in der Medienindustrie sehr viel gründlicher hinterfragt werden, als das in den vergangenen Gründerjahren der Internet-Welt der Fall war. Generell werden die Services zunehmend von Konsumenten der Inhalte bezahlt werden müssen. Dennoch bieten sich genug Modelle, die dem Kunden echten Nutzen bringen und für die die Kunden dann auch bereit sind zu zahlen. Dazu müssen aber noch einige Hausaufgaben bei der IT-Systemunterstützung gemacht werden, wobei die Elemente Billing, Digital Rights Management und CRM die vordringlichsten sind und ohne die neuen Möglichkeiten wie UTMS nicht effektiv betrieben werden können.

Bertelsmann mediaSystems ist der Service-Provider mit den größten Erfahrungen in dem Konvergenzbereich aus Medien, Telekommunikation und Informationstechnologie und somit der Treiber, der den Bertelsmann-Konzern für die Zukunft der Medienindustrie gerüstet hat. Mit diesem Konvergenz-Know-how unterstützt Bertelsmann mediaSystems das Geschäft von Kunden aus allen Branchen. 


\section{Literatur}

[Rayp99] Rayport, Jeffrey F.; Sviokla, John J. : "Exploting the virtual value chain.” In: Tapscott, Don (Hrsg.): Creating value in the network economy. Harvard Business Review, Boston, 1999, Seiten 35 - 54 .

[VonB97] "Zukunft Multimedia - Grundlagen, Märkte und Perspektiven in Deutschland." Hrsg. Von Booz, Allen \& Hamilton. 4 Aufl. Institut für Medienentwicklung und Kommunikation in der Verlagsgruppe Frankfurter Allgemeine Zeitung, Frankfurt am Main, 1997.

[Wolf99] Wolf, Micheal J.: "The entertainment economy - how mega-media forces are transforming our lives.” Times Books Random House, New York, 1999.

[Schw99] Schwartz, Evan I.: „Digital Darwinism - seven breakthrough business strategies for surviving in the cutthroat Web economy."Broadway Books Random House, New York, 1999.

[Nais86] Naisbitt, John: "Megatrends - 10 Perspektiven, die unser Leben verändern werden." 6. Aufl., Hestia Verlag, Bayreuth, 1986.

[Choi97] Choi, Soon-Yong; Stahl, Dale O.; Whinston, Andrew B.: "The economics of electronic Eommerce.” Macmillan Technical Publishing, Indianapolis, 1997.

[Hage97] Hagel, John III; Armstrong, Arthur: „Net gain - expanding markets through virtual communities.“ Harvard Business School Press, Boston, 1997.

[Cusu98] Cusumano, Michael A.: "Competing on internet time - lessons from Netscape and its battle with Microsoft." The free press, New York 1998. 